[0212-7199 (2003) 20: 4; pp 201-205] ANALES DE MEDICINA INTERNA Copyright $@ 2003$ ARAN EDICIONES, S.L.

AN. MED. INTERNA (Madrid) Vol. 20, N. ${ }^{\circ} 4$, pp. 201-205, 2003

\title{
Regulación del tono bronquial en la enfermedad pulmonar obstructiva crónica (EPOC): papel de los receptores muscarínicos
}

\author{
F. ABAD SANTOS, J. NOVALBOS, S. GALLEGO SANDÍN, Mª A. GÁLVEZ \\ MÚGICA
}

Servicio de Farmacología Clínica. Hospital Universitario de La Princesa. Madrid

REGULATION OF BRONCHIAL TONE IN CHRONIC OBSTRUCTIVE PULMONARY DISEASE (COPD): ROLE OF MUSCARINIC RECEPTORS

\section{RESUMEN}

Los fármacos anticolinérgicos son especialmente útiles en el tratamiento de la EPOC ya que el tono colinérgico vagal parece ser el único componente reversible del estrechamiento de la vía aérea en esta enfermedad, a diferencia de lo que ocurre en el asma. Estos fármacos bloquean los receptores muscarínicos del músculo liso de las vías aéreas y de las glándulas submucosas. En las vías aéreas en humanos se han identificado 3 subtipos de receptores muscarínicos que parecen tener diferentes funciones fisiológicas. Los receptores $\mathrm{M}_{1}$ de los ganglios parasimpáticos facilitan la neurotransmisión colinérgica. Los receptores $\mathrm{M}_{3}$ del músculo liso de las vías aéreas y de las glándulas median la broncoconstricción y la secreción de moco. Los receptores $\mathbf{M}_{2}$ de las terminaciones nerviosas colinérgicas inhiben la liberación de acetilcolina y por tanto actúan como retroalimentación inhibidora (autorreceptores). El bromuro de ipratropio es un antagonista muscarínico no selectivo que bloquea los receptores $\mathrm{M}_{1}, \mathrm{M}_{2}$ y $\mathrm{M}_{3}$. El tiotropio es un antagonista muscarínico nuevo, potente y de larga duración que posee selectividad cinética por los receptores $\mathrm{M}_{1} \mathrm{y}$ $\mathrm{M}_{3}$ ya que se disocia rápidamente de los receptores $\mathrm{M}_{2}$. El tiotropio administrado una vez al día por vía inhalatoria es un broncodilatador seguro y eficaz, útil como tratamiento de mantenimiento de primera línea para la EPOC.

PALABRAS CLAVE: EPOC. Receptores muscarínicos. Ipratropio. Tiotropio.

\begin{abstract}
Anticholinergic agents have proved to be of particular value in the treatment of COPD, as vagal cholinergic tone appears to be the only reversible component of airway narrowing, opposite to what happens in asthma. Anticholinergics block muscarinic receptors on airway smooth muscle an submucosal gland cells. Three subtypes of muscarinic receptors have been demonstrated in human airways. $M_{1}$ receptors in parasympathectic ganglia facilitate cholinergic neurotransmision. $M$ receptors on airway smooth muscle cells and glands mediate bronchoconstriction and mucus secretion. $M_{2}$ receptors at cholinergic nerve endings inhibit the release of acetylcholine and therefore act as feedback inhibitory receptors (autoreceptors). Ipratropium bromide is a nonselective muscarinic antagonist and therefore blocks $M_{1}, M_{2}$ and $M_{3}$ receptors. Tiotropium is a novel, potent, and long-lasting muscarinic antagonist that has a kinetic selectivity for $M_{1}$ and $M_{3}$ receptors because it dissociated very quickly from $M_{2}$ receptors. Once-daily inhaled tiotropium is a safe and effective bronchodilator useful as a first-line maintenance therapy in COPD.
\end{abstract}

KEY WORDS: COPD. Muscarinic receptors. Ipratropium. Tiotropium.

Abad Santos F, Novalbos J, Gallego Sandín S, Gálvez Múgica MA. Regulación del tono bronquial en la enfermedad pulmonar obstructiva crónica (EPOC): papel de los receptores muscarínicos. An Med Interna (Madrid) 2003; 20: 201-205.

\section{SISTEMA NERVIOSO AUTÓNOMO EN EL APARATO RESPIRATORIO}

El sistema nervioso parasimpático desempeña un papel esencial en la regulación del tono bronquial, ya que la principal inervación vegetativa de las vías aéreas en la especie humana es de tipo parasimpático. Las fibras eferentes vagales preganglionares entran en el pulmón a través de los hilios, viajan a lo largo de las vías aéreas y terminan en los ganglios parasimpáticos de las paredes de los bronquios, con predominio en los bronquios grandes y medianos (1-3). Las fibras posgangliónicas cortas van desde estos ganglios a inervar la musculatura lisa de las vías aéreas, las glándulas submucosas y las estructuras vasculares. La estimulación del nervio vago en animales produce la liberación de acetilcolina de las terminaciones nerviosas que activa los receptores muscarínicos del músculo liso y de las glándulas produciendo broncoconstricción y secreción de moco (2). La inervación colinérgica es

Trabajo aceptado: 11 de septiembre de 2002

Correspondencia: Francisco Abad Santos. Servicio de Farmacología Clínica. Hospital Universitario de la Princesa. C/ Diego de León 62. 28006 Madrid. e-mail: fabad@hlpr.insalud.es 
mayor en las vías aéreas grandes que en las periféricas, aunque en humanos se han encontrado receptores muscarínicos tanto en las vías aéreas grandes como en las pequeñas (4).

En condiciones normales existe una baja actividad tónica basal en la musculatura bronquial que depende del tono colinérgico. La actividad parasimpática aumenta a través de reflejos neurales vagales broncoconstrictores, desencadenados por la estimulación de terminaciones sensoriales próximas a las células epiteliales (receptores de irritantes). Los estímulos desencadenantes pueden ser diversos: inhalación de partículas, gases, aerosoles, aire frío o caliente, etc. Tanto en el asma como en la bronquitis crónica existe una hiperreactividad de los bronquios a estos estímulos, que puede deberse a un estado de hiperexcitabilidad a cualquier nivel del arco reflejo vagal (3).

Los nervios simpáticos inervan vasos sanguíneos y glándulas; la noradrenalina liberada causa constricción de los primeros e inhibe la secreción de las últimas. En contra de lo que se creía anteriormente, no existe inervación simpática de la musculatura lisa bronquial y todos los efectos "simpáticos" se deben a las catecolaminas circulantes (5). La mayoría de los receptores adrenérgicos de las vías aéreas son de tipo $\beta_{2}$, aunque en los alvéolos también hay receptores $\beta_{1}$. Al contrario de lo que ocurre con los receptores muscarínicos, los receptores $\beta_{2}$ son más frecuentes en las vías aéreas más pequeñas. La estimulación de los receptores $\beta$-adrenérgicos de las vías aéreas provoca relajación de la musculatura lisa, inhibición de la liberación de mediadores de las células cebadas e incremento del aclaramiento mucociliar.

Por lo tanto, los nervios colinérgicos constituyen la vía broncoconstrictora dominante en las vías aéreas en humanos y animales (1). La broncoconstricción producida por estimulación vagal cumple una función fisiológica ya que el aumento del tono de las vías aéreas ayuda a prevenir el colapso de aquellas vías aéreas que no tienen cartílago y mejora el tono de las vías cartilaginosas. Aunque la broncoconstricción produce obstrucción de la vía aérea, es necesario un pequeño aumento del tono del músculo para mantener las vías aéreas permeables, especialmente para evitar que se colapsen durante la espiración. No obstante, un tono vagal excesivo puede ser responsable de al menos parte de la obstrucción de la vía aérea que se observa en algunos pacientes (6).

\section{SUBTIPOS DE RECEPTORES MUSCARÍNICOS}

Se conocen 5 subtipos de receptores muscarínicos, de los cuales los 3 primeros, $\mathbf{M}_{1}, \mathbf{M}_{2}$ y $\mathbf{M}_{3}$, están presentes en las vías aéreas en humanos y parecen tener diferentes funciones fisiológicas (Tabla I) $(1,2,6,7)$. En estudios farmacológicos se ha visto que el músculo liso de las vías aéreas contiene receptores $\mathrm{M}_{2} \mathrm{y}$ $\mathbf{M}_{3}$, las glándulas receptores $\mathrm{M}_{1}$ y $\mathrm{M}_{3}$ en una proporción 1:2 y las paredes alveolares receptores $M_{1}(4)$, aunque algunos autores también han encontrado receptores $\mathbf{M}_{2}$ en las glándulas (2).

Las fibras vagales activan los receptores nicotínicos y muscarínicos $\mathrm{M}_{1}$ de los ganglios parasimpáticos localizados en la pared de las vías aéreas; cuando se estimulan los receptores $\mathrm{M}_{1}$ de las fibras posganglionares cortas, éstas transmiten el estímulo nervioso hasta el músculo liso y las glándulas mucosas donde liberan acetilcolina que actúa sobre los receptores $\mathrm{M}_{3}$. Estos receptores $\mathrm{M}_{1}$ de los ganglios son inhibidos por los receptores $\beta$-adrenérgicos. El antagonismo de estos receptores $\beta$ con fármacos $\beta$-bloqueantes puede producir una
TABLA I

TIPO S DE RECEPTO RES M USCARÍNICOS PRESENTES EN LOS PULM O NES.

(Adaptada de W hite, 1995 (2) y Campbell, 2000 (6))

\begin{tabular}{|c|c|c|}
\hline Receptor & Localización & Acción \\
\hline$M_{1}$ & $\begin{array}{l}\text { Ganglios parasimpáticos } \\
\text { Glándulas mucosas } \\
\text { Pared alveolar }\end{array}$ & $\begin{array}{l}\text { Facilita la transmisión sináptica } \\
\text { Aumenta la secreción } \\
?\end{array}$ \\
\hline$M_{2}$ & $\begin{array}{l}\text { M úsculo liso bronquial } \\
\text { Periferia pulmonar }\end{array}$ & $\begin{array}{l}\text { Autorreceptor: inhibe la libera- } \\
\text { ción de acetilcolina } \\
?\end{array}$ \\
\hline$M_{3}$ & $\begin{array}{l}\text { M úsculo liso bronquial } \\
\text { Glándulas mucosas }\end{array}$ & $\begin{array}{l}\text { Broncoconstricción } \\
\text { Aumento de la secreción }\end{array}$ \\
\hline
\end{tabular}

actividad muscarínica sin oposición en las vías aéreas, que da lugar a broncospasmo. Por este motivo, los fármacos antimuscarínicos como la atropina o el ipratropio son los más eficaces para resolver el broncospasmo producido por $\beta$-bloqueantes (6). Existen receptores $M_{1}$ en otros sitios del pulmón como la pared alveolar, pero no se sabe qué función desempeñan puesto que no hay inervación colinérgica en el parénquima pulmonar (1). Algunos estudios indican que el antagonismo selectivo de los receptores $M_{1}$ puede producir broncodilatación (6).

Los receptores $M_{3}$ están localizados en el músculo liso de las vías aéreas y de las glándulas submucosas y son los que producen el efecto parasimpático de broncoconstricción y aumento de las secreciones que cambian a una consistencia más acuosa (8). El antagonismo de este receptor es el principal responsable del efecto broncodilatador producido por los fármacos antagonistas muscarínicos. El receptor $M_{3}$ se encuentra en abundancia en el músculo liso de las vías aéreas grandes (7), pero también existe en las vías aéreas pequeñas y distribuido por todo el pulmón (9), aunque su papel a este nivel es desconocido, especialmente porque no hay inervación parasimpática en estas áreas (6).

Los receptores $M_{2}$ se encuentran en la porción terminal del nervio; son "autorreceptores" que median efectos de retroalimentación negativos de tal modo que disminuyen la liberación de acetilcolina de los nervios colinérgicos (7). El bloqueo de este receptor aumenta la liberación de acetilcolina y puede producir broncoconstricción (1).

\section{IMPLICACIONES DEL SISTEMA PARASIMPÁTICO EN LA PATOLOGÍA RESPIRATORIA}

En la etiopatogenia de la hiperreactividad bronquial puede estar implicada una disfunción en la regulación autónoma del tono bronquial. Se ha visto que los individuos asmáticos presentan una elevada actividad tónica del sistema parasimpático broncoconstrictor, un aumento de la actividad $\alpha$-adrenérgica, un aumento de la actividad excitadora no adrenérgica no colinérgica (taquicininas o péptido relacionado con el gen de la calcitonina) y una disminución del sistema simpático broncodilatador $\beta$ y del sistema inhibidor no adrenérgico no colinérgico (VIP). Se supone que este desequilibrio vegetativo puede ser consecuencia de la estimulación por bradicinina de las ter- 
minaciones nerviosas desprotegidas por un epitelio lesionado, lo que desencadenaría un reflejo vagal broncoconstrictor mediado por acetilcolina (3).

En los pacientes con enfermedad pulmonar obstructiva crónica (EPOC), el sistema colinérgico está intacto porque cuando estos pacientes respiran aire frío (efecto broncoconstrictor mediado por mecanismos colinérgicos) se produce un aumento de las resistencias que revierte con ipratropio (10).

En conclusión, el principal componente reversible de la EPOC es el tono colinérgico vagal. Por el contrario, en el asma, aunque existe un aumento del tono colinérgico de las vías aéreas, hay otros mecanismos broncoconstrictores que pueden desempeñar un papel más relevante, como los mediadores inflamatorios histamina y leucotrienos (1).

\section{EFECTOS EN EL APARATO RESPIRATORIO DE LOS} ANTAGONISTAS DE LOS RECEPTORES MUSCARÍNICOS

Los fármacos anticolinérgicos bloquean de forma competitiva el efecto de la acetilcolina sobre los receptores de la musculatura lisa del árbol bronquial. La eficacia broncodilatadora va a depender del grado en el que el reflejo colinérgico broncoconstrictor contribuye al broncospasmo que presenta cada paciente concreto. La distribución predominantemente central de los receptores muscarínicos en las vías aéreas constituye una limitación a la eficacia de estos fármacos. La broncodilatación se produce sobre todo en los bronquios grandes y medianos, pero es menor en los bronquiolos, lo que implica una gran variabilidad en la respuesta de cada sujeto (6). En algunos estudios se ha comprobado que los fármacos antimuscarínicos también producen relajación de las vías aéreas más periféricas, aunque en menor medida que la producida por fármacos $\beta$-agonistas (11).

La actividad anticolinérgica se manifiesta con independencia de cual sea la causa de aumento del tono vagal, por lo que son útiles tanto en asma como en EPOC. Sin embargo, el tono colinérgico es mayor en EPOC que en asma por lo que son más útiles para el tratamiento de la primera enfermedad (3).

Desde hace muchos años se sabe que los alcaloides de la belladona inhiben las secreciones de la nariz, la boca, la faringe y los bronquios, y, por lo tanto, secan las membranas mucosas del tracto respiratorio. Este efecto es especialmente marcado cuando la secreción es excesiva y constituye la base para la utilización de atropina y escopolamina como medicaciones preanestésicas. Además, estos fármacos reducen la incidencia de laringospasmo durante la anestesia general, lo que se puede deber a la inhibición de las secreciones respiratorias que pueden precipitar el broncospasmo reflejo. Sin embargo, la disminución de las secreciones mucosas y del aclaramiento mucociliar son efectos indeseables en pacientes con enfermedades de las vías aéreas (12). Por otro lado, la atropina produce relajación de la musculatura bronquial, debido principalmente al bloqueo de los receptores $\mathrm{M}_{3}$. También inhibe la broncoconstricción inducida por mediadores de la inflamación como histamina, bradicinina y eicosanoides, posiblemente porque los eferentes parasimpáticos participan en los reflejos bronquiales que provocan estos agentes. La capacidad de los fármacos anticolinérgicos para bloquear los efectos broncoconstrictores indirectos de los mediadores inflamatorios justifica su utilización durante enfermedades como el asma o la enfermedad pulmonar obstructiva crónica.

El grado de broncodilatación que consiguen estos fármacos refleja el nivel de tono parasimpático basal. En sujetos normales, la inhalación de estos fármacos protege completamente frente a la broncoconstricción producida por la inhalación subsecuente de sustancias como dióxido de sulfuro, ozono o humo de tabaco. No obstante, en los pacientes con asma o hiperreactividad bronquial el efecto protector es menor. Aunque estos fármacos producen una marcada reducción de la sensibilidad a metacolina en pacientes asmáticos, producen una inhibición menor de la provocación con histamina, bradicinina, prostaglandina $\mathrm{F}_{2 \alpha}$, y protegen muy poco frente a la broncoconstricción inducida por serotonina o leucotrienos (12).

El mecanismo por el cual los antagonistas muscarínicos ayudan a aliviar la disnea de los pacientes con enfermedad pulmonar no se conoce completamente, ya que relajan poco el músculo liso de las vías aéreas más distales. No obstante, algunas personas presentan un aumento importante de la capacidad vital al ser tratadas con fármacos antimuscarínicos (6). Una posible explicación podría ser que produzcan broncodilatación importante de las vías aéreas periféricas, pero esto no concuerda con los datos disponibles en la literatura, por lo que es posible que actúen sobre receptores localizados en otros sitios dentro del pulmón (6).

El tratamiento a largo plazo con antagonistas muscarínicos por vía inhalatoria es bien tolerado, al igual que los $\beta$-agonistas inhalados, pero a diferencia de éstos su eficacia se mantiene a largo plazo ya que no se aprecia el desarrollo de tolerancia o taquifilaxia (13).

Desde hace años se ha discutido si los antagonistas muscarínicos tienen efecto antiinflamatorio. En algunos experimentos parece que los antagonistas muscarínicos pueden inhibir la liberación de histamina por las células cebadas, un efecto mediado por receptores $\mathrm{M}_{1}$, aunque no se ha confirmado en todos los estudios (14). En un estudio reciente, la acetilcolina, a través de la activación de los receptores $\mathrm{M}_{3}$, induce en los macrófagos alveolares la liberación de sustancias quimiotácticas derivadas de la lipooxigenasa que atraen neutrófilos, eosinófilos y monocitos (15); con lo cual los antimuscarínicos sí que reducirían la inflamación.

Aunque en algunos estudios se ha visto que los antimuscarínicos cuaternarios por vía inhalada pueden reducir el volumen de esputo sin alterar su viscosidad (16), el efecto sobre las secreciones bronquiales es inferior al producido por la atropina y en otros estudios no ha sido significativamente distinto del placebo $(17,18)$. Es interesante que la reducción del volumen de esputo no sigue el curso temporal del efecto broncodilatador: empieza a las 3 semanas y disminuye progresivamente hasta las 7 semanas de tratamiento, lo que indica que no se produce simplemente por el bloqueo agudo de la secreción inducida por acetilcolina (14). También se debe destacar que los antimuscarínicos cuaternarios inhalados no reducen el aclaramiento mucociliar $(17,18)$.

\section{USO DE ANTAGONISTAS MUSCARÍNICOS EN LA EPOC}

Los fármacos anticolinérgicos son especialmente útiles en el tratamiento de la EPOC ya que el tono colinérgico vagal parece ser el único componente reversible del estrechamiento de la vía aérea en esta enfermedad (19).

La EPOC muchas veces es manejada en la práctica clínica 
como un asma poco respondedora, aunque estas 2 enfermedades son muy diferentes en cuanto a los mecanismos celulares y mediadores inflamatorios implicados, y en cuanto a la respuesta al tratamiento. No obstante, alrededor del 10\% de los pacientes con EPOC también padecen asma (8). Ambas enfermedades se caracterizan por una obstrucción al flujo de la vía aérea y un proceso inflamatorio crónico persistente, aunque la naturaleza de la inflamación difiere marcadamente entre ellas. Mientras que en el asma las células más implicadas son los eosinófilos y las células cebadas, en la EPOC predominan los neutrófilos y los macrófagos. Mientras que en el asma están afectadas todas las vías aéreas sin implicar al parénquima, en la EPOC la lesión es mayor en las vías aéreas periféricas, existe destrucción de parénquima, y hay más fibrosis y secreción de moco. A diferencia del asma, en la EPOC no suele existir hiperreactividad de la vía aérea, aunque puede existir un aumento de la respuesta a broncoconstrictores como agonistas colinérgicos o histamina. Además, los pacientes con EPOC no presentan broncoconstricción con estímulos indirectos como el ejercicio o la bradicinina (8).

En el asma se han implicado más de 50 mediadores inflamatorios y los reflejos colinérgicos pueden ser activados por algunos de estos mediadores como las cininas. Los agonistas $\beta$-adrenérgicos son los broncodilatadores más eficaces en el asma porque actúan como antagonistas funcionales y contrarrestan el efecto broncoconstrictor de múltiples mediadores. Por el contrario, en la EPOC se liberan pocos mediadores inflamatorios y el tono colinérgico es probablemente el único componente reversible (8). Por este motivo, los fármacos anticolinérgicos son más eficaces en la EPOC que los $\beta$-agonistas.

El asma es la principal enfermedad obstructiva de la vía aérea que se produce principalmente a nivel de las vías aéreas pequeñas. Por este motivo, los $\beta$-agonistas son más eficaces en el asma, ya que inhiben por igual la constricción de las vías aéreas grandes y pequeñas, a diferencia de los fármacos antimuscarínicos que actúan principalmente sobre la constricción de las vías aéreas grandes. No obstante, los anticolinérgicos pueden proporcionar un beneficio adicional asociados a los $\beta_{2}$-adrenérgicos, especialmente en el tratamiento del asma aguda tanto en niños y adolescentes como en adultos, ya que mejoran la función pulmonar y reducen la necesidad de ingreso hospitalario $(20,21)$. Por el contrario, la EPOC se caracteriza por la destrucción de las paredes de las vías aéreas, lo que produce obstrucción irreversible, y, en este caso, la relajación del tono vagal puede todavía producir una mejoría del calibre de las vías aéreas (2).

Actualmente, el fármaco antimuscarínico ipratropio constituye el tratamiento de primera línea para los pacientes con EPOC ya que es más eficaz que los $\beta$-agonistas porque consigue una mayor mejoría sintomática y una reducción de las exacerbaciones $(13,14)$. En los pacientes con EPOC, la combinación de un antagonista muscarínico con un $\beta$-agonista produce un mayor efecto broncodilatador que cada fármaco por separado (22), aunque la reducción de las exacerbaciones parece deberse a la presencia del antagonista muscarínico (14). Por el contrario, en el asma los antimuscarínicos no son tan eficaces como los $\beta$-agonistas y pueden no aportar ningún beneficio cuando se administran dosis máximas de $\beta$-agonistas. No obstante, en algunos pacientes la adición de ipratropio a dosis bajas de $\beta$-agonistas puede proporcionar una mayor broncodilatación sin los efectos adversos que se producirían si se aumentase la dosis de $\beta$-agonistas (6).

Como consecuencia de lo expuesto anteriormente, es razo- nable que en múltiples recomendaciones de tratamiento se considere a los antimuscarínicos inhalados como el tratamiento de primera línea para la EPOC, la bronquitis crónica y el enfisema $(14,23)$. Sin embargo, en el asma constituyen un tratamiento de segunda línea después de los agonistas $\beta_{2}$-adrenérgicos.

\section{TIPOS DE ANTAGONISTAS MUSCARÍNICOS}

El fármaco antimuscarínico ideal para tratar las enfermedades de las vías aéreas debería bloquear los receptores muscarínicos $\mathrm{M}_{1}$ y los $\mathrm{M}_{3}$ del músculo liso bronquial, sin bloquear los receptores $\mathbf{M}_{2}$. Sin embargo, los fármacos disponibles actualmente no son selectivos. Bloquean el receptor $\mathrm{M}_{3}$ produciendo broncodilatación, pero también sequedad del moco. También bloquean el receptor $\mathbf{M}_{2}$ lo que puede dar lugar a obstrucción por desinhibición de la secreción de acetilcolina; sin embargo, no existen evidencias de que el bloqueo de los receptores $\mathrm{M}_{2}$ con los fármacos actuales sea perjudicial porque también bloquean los receptores $\mathrm{M}_{1}$ y $\mathrm{M}_{3}(6)$.

Los antagonistas muscarínicos derivados de amonio cuaternario, como el bromuro de ipratropio, presentan una serie de ventajas frente a los derivados de amonio terciario como la atropina, por lo que son los que se utilizan en patología respiratoria. La principal ventaja de los derivados cuaternarios es que no producen efectos sistémicos cuando se administran por vía inhalatoria porque, al ser muy polares, no se absorben, y no producen efectos sobre el SNC porque no pasan la barrera hematoencefálica (24). Además, apenas producen efecto inhibidor del aclaramiento mucociliar por lo que evitan la acumulación de secreciones que se produce con atropina (12).

El bromuro de tiotropio es un nuevo antagonista muscarínico derivado de amonio cuaternario, parecido al bromuro de ipratropio, que se va a comercializar próximamente y que presenta una gran ventaja: su efecto broncodilatador se mantiene más de $24 \mathrm{~h}$ lo que permite su administración en una dosis al día, a diferencia del ipratropio que se debe administrar cada 6 h (24). Esto facilitará el cumplimiento terapéutico de los pacientes y el mejor control de la sintomatología durante todo el día, incluido durante el reposo nocturno. En los estudios in

\section{TABLA ॥}

COM PARACIÓ N DE LAS CARACTERÍSTICAS DE BRO M URO DE IPRATRO PIO Y BRO MURO DE TIOTRO PIO. (Adaptada de Barnes, 2000) (19)

\begin{tabular}{lcc}
\hline Características & Ipratropio & Tiotropio \\
\hline Constante de disociación $(\mathrm{nM})^{*}$ & & \\
M 1 & 0,18 & 0,04 \\
M 2 & 0,20 & 0,02 \\
M 3 & 0,20 & 0,01
\end{tabular}

Vida media de disociación del receptor (h)

$\begin{array}{ccc}\text { M } 1 & 0,11 & 14,6 \\ \text { M2 } & 0,035 & 3,6 \\ \text { M } 3 & 0,26 & 34,7\end{array}$

Duración del efecto
$6 \mathrm{~h}$

$24 \mathrm{~h}$

4,6 34,7 (intervalo de dosificación) 
vitro, el tiotropio es 6-10 veces más potente que el ipratropio, su efecto se produce más lentamente (se alcanza el 50\% del efecto máximo a los 35 min frente a los 8 min de ipratropio) pero dura 6-7 veces más (el 50\% del efecto desaparece a los $540 \mathrm{~min}$ frente a $81 \mathrm{~min}$ con ipratropio) $(19,25)$.

El ipratropio bloquea los subtipos de receptores muscarínicos $\mathbf{M}_{1}, \mathrm{M}_{2}$ y $\mathrm{M}_{3}$ (Tabla II). Es posible que el bloqueo que produce de los autorreceptores $\mathrm{M}_{2}$ en los nervios colinérgicos incremente la liberación de acetilcolina y reduzca la eficacia de su antagonismo en los receptores $\mathrm{M}_{3}$ del músculo liso. Por el contrario, el tiotropio muestra selectividad por $M_{1}$ y $M_{3}$ ya que su velocidad de disociación de estos receptores es muy lenta ( $35 \mathrm{~h}$ si se compara con los 16 min de ipratropio) y se disocia rápidamente de los receptores $M_{2}(3,6$ h) $(14,25)$. Este es un tipo de selectividad cinética por los receptores $M_{3}\left(\mathrm{y} \mathrm{M}_{1}\right)$ con respecto a los $\mathrm{M}_{2}$. De hecho, los estudios autorradiográficos en humanos han demostrado que se marcan predomi- nantemente los receptores $\mathrm{M}_{1} \mathrm{y}_{3}$ (26). Por estas propiedades, el efecto del tiotropio presenta una duración mucho más prolongada que el ipratropio.

En pacientes con EPOC, el tiotropio produce una broncodilatación significativa que es dependiente de la dosis en los estudios de dosis única y persiste durante más de 24 h (27). En los estudios de dosis múltiple produce una mejoría persistente de la espirometría, pero sin relación clara con la dosis (28), lo que puede estar relacionado con la lenta disociación de los receptores muscarínicos. En los estudios de 3 a 12 meses de duración con una dosis de $18 \mathrm{mcg}$ una vez al día se consigue una mejoría persistente que incluso es significativamente superior a la producida por ipratropio $(29,30)$. Además de mejorar los parámetros espirométricos, mejora la sintomatología, reduce la necesidad de medicación de rescate y es muy bien tolerado. El único efecto adverso relacionado con el tratamiento es la sequedad de boca, que se ha presentado en $9-15 \%$ de los pacientes tratados con tio-

\section{Bibliografía}

1. Barnes PJ, Belvisi MG, Mak JC, Haddad EB, O'Connor B. Tiotropium bromide (Ba $679 \mathrm{BR}$ ), a novel long-acting muscarinic antagonist for the treatment of obstructive airways disease. Life Sci 1995; 56: 853-9.

2. White MV. Muscarinic receptors in human airways. J Allergy Clin Immunol 1995; 95: 1065-8.

3. Hurlé MA. Fármacos antiasmáticos y broncodilatadores. En: Flórez J, Armijo JA, Mediavilla A, dir. Farmacología humana, $3^{a}$ ed. Barcelona: Masson, 1997; 705-21.

4. Mark JCW, Barnes PJ. Autoradiographic visualization of muscarinic receptors subtypes in human and guinea pig lung. Am Rev Respir Dis 1990; 141: 1559-68.

5. Rang HP, Dale MM. Sistema respiratorio. En: Rang HP, Dale MM. Farmacología. Madrid: Alhambra Longman, 1992; 430-50.

6. Campbell SC. Clinical aspects of inhaled anticholinergic therapy. Respir Care 2000; 45: 864-7.

7. Emala CW, Aryana A, Levine MA, Yasuda RP, Satkus SA, Wolfe BB, et al. Expression of muscarinic receptor subtypes and M2-muscarinic inhibition of adenylyl cyclase in lung. Am J Physiol 1995; 268: L101-7.

8. Barnes PJ. Mechanisms in COPD: differences from asthma. Chest 2000; 117 (Supl. 2): 10S-14S.

9. Pistelli R, Patalano F, Liberatore SM, Incalzi RA, Ciappi G. Selectivity of anticholinergic drugs on central and peripheral airways in normal subjects. Eur J Respir Dis 1983; 128 (Supl.): 499-502.

10. Calverley PM. The future for tiotropium. Chest 2000; 117 (Supl. 2): 67S-69S.

11. Haddad EB, Mak JC, Belvisi MG, Nishikawa M, Rousell J, Barnes PJ Muscarinic and $\beta$-adrenergic receptor expression in peripheral lung from normal and asthmatic patients. Am J Physiol 1996; 270: L947-53.

12. Brown $\mathrm{JH}$, Taylor P. Muscarinic receptors agonists and antagonists. En: Hardman JG, Limbird LE, Gilman AG, eds. Goodman \& Gilman's The Pharmacologic Basis of Therapeutics, $10^{\mathrm{a}}$ edición. Nueva York: McGrawHill, 2001: 155-73.

13. Tashkin DP, Ashutosh K, Bleecker ER, Britt EJ, Cugell DW, Cummiskey $\mathrm{JM}$, et al. Comparison of the anticholinergic bronchodilator ipratropium bromide with metaproterenol in chronic obstructive pulmonary disease. A 90-day multi-center study. Am J Med 1986; 81 (5A): 81-90.

14. Disse B. Antimuscarinic treatment for lung diseases: from research to clinical practice. Life Sci 2001; 68: 2557-64.

15. Sato E, Koyama S, Okubo Y, Kubo K, Sekiguchi M. Acetylcholine stimulates alveolar macrophages to release inflammatory cell chemotactic activity. Am J Physiol 1998; 274: L970-9.

16. Ghafouri MA, Patil KD, Kass I. Sputum changes associated with the use of ipratropium bromide. Chest 1984; 86: 387-93.

17. Taylor RG, Pavia D, Agnew JE, Lopez-Vidriero MT, Newman SP, Lennard-Jones T et al. Effect of four weeks' high dose ipratropium bromide tre- atment on lung mucociliary clearance. Thorax 1986; 41: 295-300.

18. Pavia D, Lopez-Vidriero MT, Agnew JE, Taylor RG, Eyre-Brook A, Lawton WA et al. Effect of four-week treatment with oxitropium bromide on lung mucociliary clearance in patients with chronic bronchitis or asthma. Respiration 1989; 55: 33-43.

19. Barnes PJ. The pharmacological properties of tiotropium. Chest 2000; 117 (Supl. 2): 63S-6S.

20. Plotnick LH, Ducharme FM. Should inhaled anticholinergics be added to $\beta 2$ agonists for treating acute childhood and adolescent asthma? A systematic review. Br Med J 1998; 317: 971-7.

21. Rodrigo G, Rodrigo C, Burschtin O. A meta-analysis of the effects of ipratropium bromide in adults with acute asthma. Am J Med 1999; 107: 363-70.

22. Campbell S. For COPD a combination of ipratropium bromide and albuterol sulfate is more effective than albuterol base. Arch Intern Med 1999; 159: 156-60.

23. BTS guidelines for the management of chronic obstructive pulmonary disease: the COPD Guideline Group of the Standards of Care Committee of the BTS. Thorax 1997; 52 (Supl. 5): S1-28.

24. Disse B, Speck GA, Rominger KL, Witek TJ Jr, Hammer R. Tiotropium (Spiriva): mechanistical considerations and clinical profile in obstructive lung disease. Life Sci 1999; 64: 457-64.

25. Takahashi T, Belvisi MG, Patel H, Ward JK, Tadjkarimi S, Yacoub MH, et al. Effect of Ba $679 \mathrm{BR}$, a novel long-acting anticholinergic agent, on cholinergic neurotransmission in guinea pig and human airways. Am J Respir Crit Care Med 1994; 150: 1640-5

26. Haddad EB, Mak JC, Barnes PJ. Characterization of $[3 \mathrm{H}] \mathrm{Ba} 679$ $\mathrm{BR}$, a slowly dissociating muscarinic antagonist, in human lung: radioligand binding and autoradiographic mapping. Mol Pharmacol 1994; 45: 899-907.

27. Maesen FP, Smeets JJ, Sledsens TJ, Wald FD, Cornelissen PJ. Tiotropium bromide, a new long-acting antimuscarinic bronchodilator: a pharmacodynamic study in patients with chronic obstructive pulmonary disease (COPD). Dutch Study Group. Eur Respir J 1995; 8: 1506-13.

28. Littner MR, Ilowite JS, Tashkin DP, Friedman M, Serby CW, Menjoge SS et al. Long-acting bronchodilation with once-daily dosing of tiotropium (Spiriva) in stable chronic obstructive pulmonary disease. Am J Respir Crit Care Med 2000; 161: 1136-42.

29. Casaburi R, Briggs DD Jr, Donohue JF, Serby CW, Menjoge SS, Witek TJ Jr. The spirometric efficacy of once-daily dosing with tiotropium in stable COPD: a 13-week multicenter trial. The US Tiotropium Study Group. Chest 2000; 118: 1294-302.

30. Van Noord JA, Bantje TA, Eland ME, Korducki L, Cornelissen PJ. A randomised controlled comparison of tiotropium and ipratropium in the treatment of chronic obstructive pulmonary disease. The Dutch Tiotropium Study Group. Thorax 2000; 55: 289-94. 